case report

\title{
Triple synchronous cancers: a medical and ethical problem
}

\author{
Lučka Debevec, Rok Cesar, Izidor Kern
}

University Clinic of Respiratory and Allergic Diseases Golnik, Golnik, Slovenia

\begin{abstract}
Background. In a patient with suspicious synchronous multiple tumours, there are limited possibilities for effective therapy. Therefore, the decision for invasive diagnostics and precise staging of tumours is questionable, especially in elderly patients suitable only for symptomatic therapy.

Case report. A 78-year-old man with hypertension and angina pectoris was admitted to the hospital due to syncope. Two primary lung tumours and a kidney tumour were detected by imaging investigation. The patient refused invasive diagnostics and left the hospital. After 19 months he was readmitted in an impaired clinical condition and subsequently died of bronchopneumonia. The autopsy revealed squamous cell carcinoma of the right upper lobe with metastases to regional lymph nodes and to the brain, small-cell carcinoma of the left upper lobe with metastases to regional lymph nodes and to the spleen, and clear-cell kidney carcinoma with multiple metastases to the lungs. All tumours were necrotizing, and therefore we assumed that any attempt at specific therapy would have been ineffective.
\end{abstract}

Conclusions. In an elderly patient with advanced lung tumors and suspicious synchronous triple cancers, the "wait and see" option can be suitable.

Key words: neoplasms, multiple primary; prognosis; ethics, medical

\section{Introduction}

Multiple synchronous cancers in the same organ or in various organs are rather unusual. Sometimes this condition is diagnosed during the staging process of the tumour, by postoperative histology of a resected organ, but it is mostly identified by autopsy. During the diagnostic procedure

\section{Received 16 April 2007}

Accepted 23 April 2007

Correspondence to: Assist. Lučka Debevec, MD, PhD, University Clinic of Respiratory and Allergic Diseases Golnik, SI-4204 Golnik, Slovenia; Phone: +386 42569 100; Fax: +386 42569 117; E-mail: lucka.debevec@ klinika-golnik.si involving the patient with suspicious multiple tumours, a dilemma appears relative to the precise staging of each of all probable tumours in a patient suitable only for symptomatic therapy.

A case of synchronous triple tumours is presented in which noninvasive diagnostic procedures were performed and suspicion of triple primary cancer was established. The patient refused invasive diagnostics and left the hospital. Fortunately, the course of the disease was rather favorable, and none of the three tumours caused symptoms requiring urgent invasive diagnostics or attempts at any specific therapies. Despite three disseminated carcinomas, the patient survived 


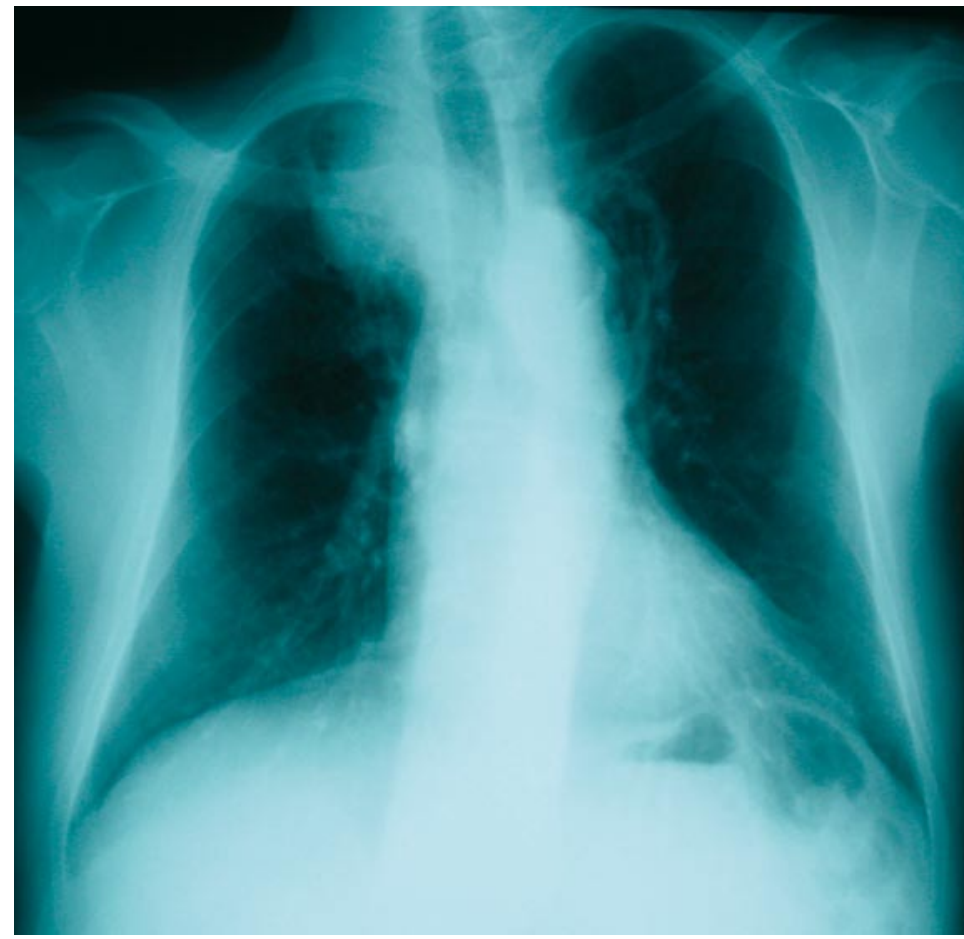

Figure 1. X-ray of the chest on the first admission (6 June 2005).

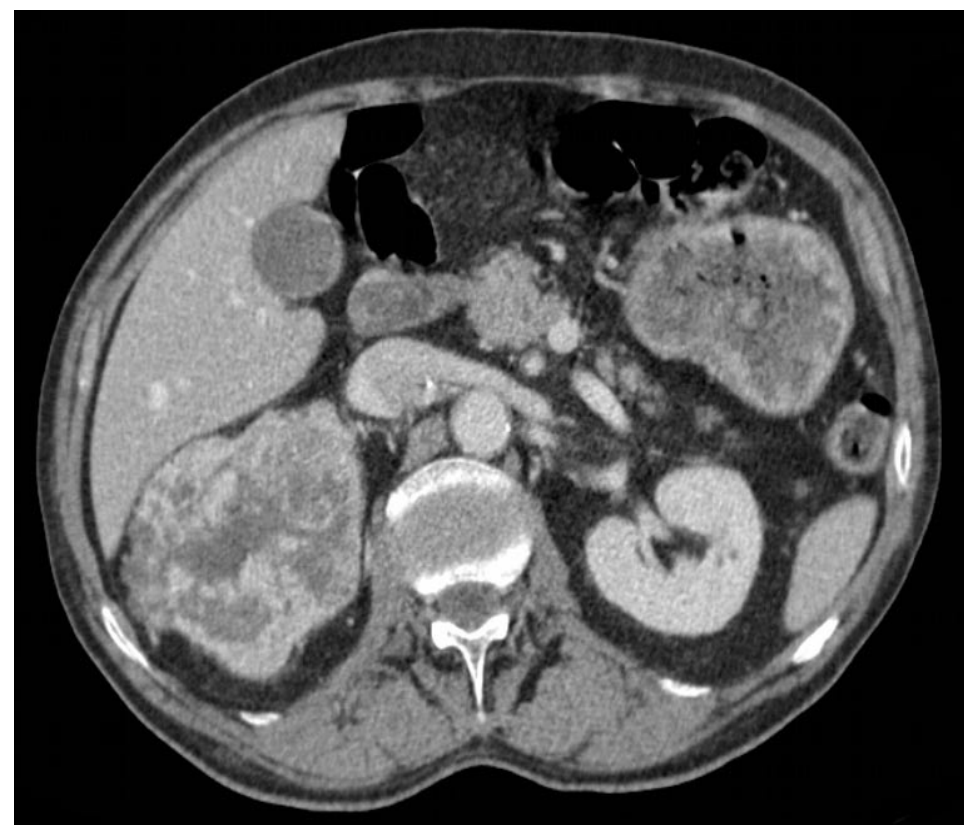

Figure 2. CT scan of the abdomen (9 June 2005). at home, i.e. without specific or symptomatic therapy needing hospitalization, for 19 months after the diagnosis of two lung tumours and a kidney tumour.

\section{Case report}

A 78-year-old man with hypertension and angina pectoris was brought to the hospital due to syncope without convulsions in June 2005. At the time of admittance he had no fever but a dry cough. Laboratory tests indicated anemia ( $\mathrm{Hb} 9.2 \mathrm{~g} / \mathrm{dL})$, leucocytosis (WBC 16.5 x $\left.10^{3} / \mu \mathrm{L}\right)$, hypokalemia (potassium $3.4 \mathrm{mmol} /$ L), slightly elevated BUN and creatinine, and low serum iron $(2.7 \mu \mathrm{mol} / \mathrm{L})$.

A CT scan of the brain showed diffuse atrophy without signs of metastasis. On the chest X-ray, tumours infiltrating in both upper lobes were visible. The lesion on the left side was excavated (Figure 1). A CT scan of the thorax and upper abdomen showed - in addition to tumour lesions in the left and right lung - also a tumour-enlarged right kidney (Figure 2). The conclusion after the im-

Radiol Oncol 2007; 41(2): 80-85. 
aging procedures was the probability of three synchronous primary tumours: a tumour in the right upper lobe infiltrating the mediastinum, a tumour in the left upper lobe with metastases to hilar and mediastinal lymph nodes, and a tumour in the right kidney.

The patient did not agree to further invasive diagnostics for verification of the tumours, and despite some persuasion he left the hospital with advice for a urological examination.

By the end of December 2006, the patient was admitted bedridden, dehydrated, somnolent, with respiratory insufficiency, anisocoria and anamnesis of frequent vomiting during the previous few days. Laboratory tests showed high inflammatory parameters (CRP $97.2 \mathrm{mg}$ ) $\left.\mathrm{dL}, \mathrm{WBC} 16.6 \times 10^{3} / \mu \mathrm{L}\right)$, and elevated BUN (50.8 mmol urea /L) and creatinine $(149 \mu \mathrm{mol} / \mathrm{L})$, but there was no anemia (hemoglobin was currently $15.9 \mathrm{~g} / \mathrm{dL}$ ).

A brain CT showed cerebral atrophy and a coliquated round lesion in the right occipital region without surrounding oedema (Figure 3). On the chest X-ray, the

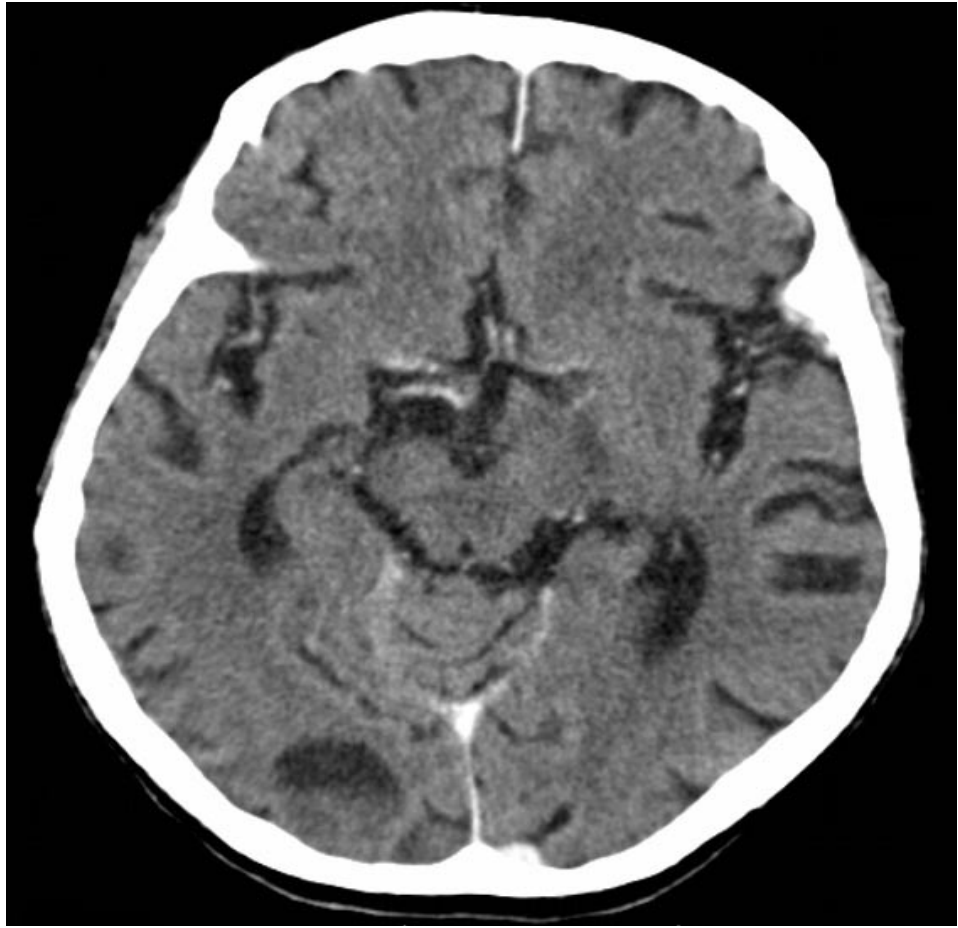

Figure 3. CT scan of the brain (28 December 2006).

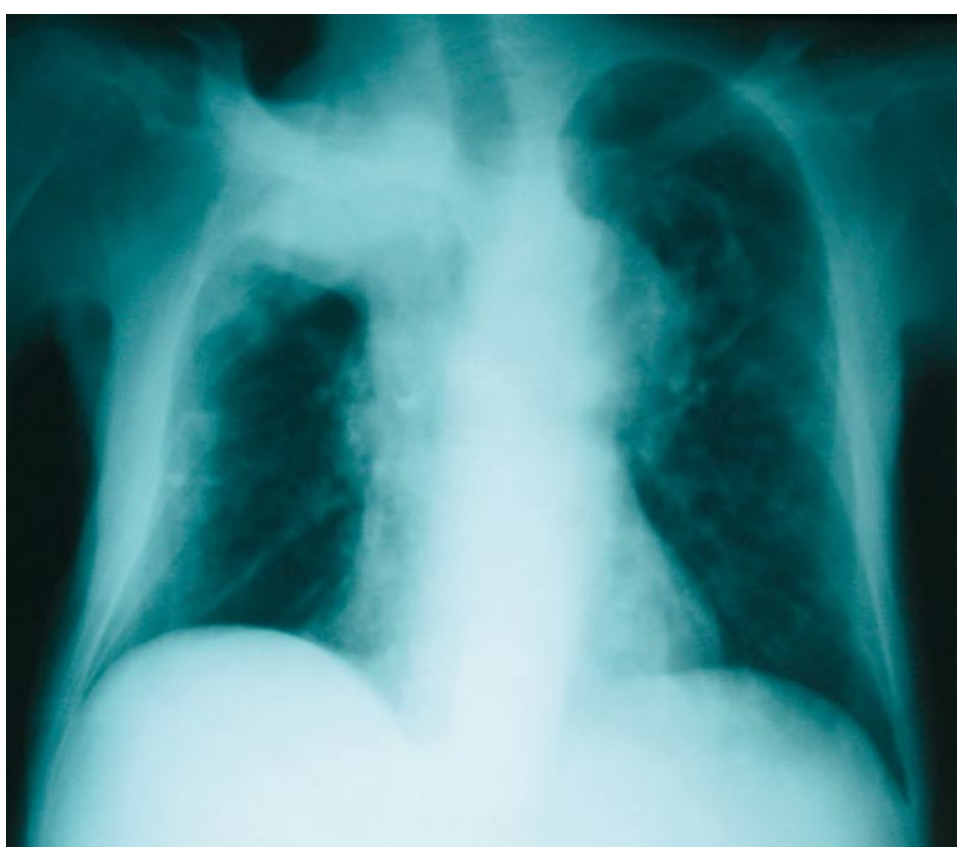

Figure 4. X-ray of the chest on the second admission (26 December 2006). 
known tumours of the upper lobes and diffuse infiltrates in both lungs appeared (Figure 4).

The patient received antibiotics and parenteral hydration, and died four days after admittance.

The autopsy revealed the excavated tumour of the left upper lobe, $7 \mathrm{~cm}$ in diameter, infiltrating the left main bronchus, to be a necrotizing small-cell carcinoma with metastases to ipsilateral hilar and ipsilateral mediastinal lymph nodes, and with necrotizing spleen metastasis of $3 \mathrm{~cm}$.

The tumour of the right upper lobe, $10 \mathrm{~cm}$ in diameter, was also necrotizing. Due to enlarged and coliquated hilar and mediastinal lymph nodes, there was an impression of tuberculosis. But histology showed a necrotizing squamous cell carcinoma of the right lung and lymph nodes, and a necrotizing metastasis of the same histology was revealed in the right occipital region of the brain.

The right kidney was entirely transformed by a $13 \mathrm{~cm}$ tumour infiltrating the renal capsule and perirenal fat tissue. Histology revealed a necrotizing well differentiated clear-cell carcinoma with multiple metastases to both lungs.

All three primary tumours as well the metastases of the brain, spleen, right hilar and mediastinal lymph nodes, were necrotizing. The cause of death was bronchopneumonia.

\section{Discussion}

In patients with two or more tumour lesions detected in various organs, one reasons that it is a primary tumour with metastases, which is statistically most probable. Synchronous triple tumours are uncommon. In the annual reports of the Cancer Registry of Slovenia it is not possible to determine the number of patients with mul- tiple cancers, since these are registered as different single tumours, i.e. cancer cases, irrespective of the number of patients. ${ }^{1}$ So, a triple cancer would be registered three times, despite involving the same patient. In the literature it is difficult to obtain data on synchronous cancers. Hamada et al. ${ }^{2}$ reported a gradual increase of triple primary cancers in Japan in the period 1994-1996. The incidence of triple cancers represented $0.81 \%$ of all autopsy cases reported.

There are interesting site combinations of triple cancers. The most frequently reported were all tumours in the same organ or organ system: lung, ${ }^{3-9}$ digestive organs, ${ }^{10-}$ 13 and urogenital organs. ${ }^{14-19}$ Lung cancer appeared in combination with tumours of two various organs: urinary bladder and esophagus, ${ }^{20}$ breast and stomach, ${ }^{21}$ pancreas and duodenum, ${ }^{22}$ and stomach and thyroid. ${ }^{2}$ Kidney cancer was seen in combination with tumours of the liver and oral floor, ${ }^{23}$ sigmoid colon and thyroid. ${ }^{24}$ On PubMed one can find articles on synchronous triple tumours, for all successfully resected ones, but mostly published in Japanese without an English abstract. Therefore, the many cases of synchronous triple cancers established in Japan raise the possibility of the influence of atomic bomb radiation on cancer incidence. Hakada et al. ${ }^{17}$ mentioned that there had been a patient with synchronous cancers of the kidney, urinary bladder and prostate exposed to the atomic bomb explosion in Hiroshima in 1945.

In our patient presented above, it is questionable whether the disease could have progressed otherwise, even with the patient's agreement to invasive diagnostics at the time of first admittance to the hospital. Probably one or both lung tumours could have been established by bronchoscopy. A needle aspiration biopsy under ultrasound guidance would have been necessary for verification of the kidney tumour. Had there been successful definition of all primary tu- 
mours, carefully dosed chemotherapy for lung cancer would parhaps have been applied. The kidney tumour would certainly not have responded to chemotherapy, and embolization of the renal artery would have been indicated, especially in the case of haematuria. Concerning the necrosis of all primary tumours and their metastases to the brain, spleen and thoracic lymph nodes, as established by the autopsy, chemotherapy would probably not have influenced survival, but certainly would have impaired the patient's quality of life. The patient decided on "wait and see" management and lived at home to the age of 80 , thus saving the physicians the dilemma of performing invasive investigation and staging of the tumours. Considering that at the time of first admittance a lack of the possibility of effective therapy was already evident, even the objection to bronchoscopy could be considered reasonable.

In conclusion, in the case of synchronous triple cancers in an elderly patient the possibilities of effective therapy are limited. Therefore, invasive diagnostics and accurate staging may not be indicated in patients with bilateral advanced primary lung tumours. A "wait and see" decision can be quite a reasonable option.

\section{References}

1. Cancer Registry of Slovenia. Cancer incidence in Slovenia 2003. Report No 45. Ljubljana: Institute of Oncology, Epidemiology and cancer Registry; 2006.

2. Hamada $Y$, Takise A, Uno D, Itoh H, Ichikawa $H$, Morishta Y. Synchronous primary triple cancers including the lung, stomach, and thyroid: a case report. Kyobu Geka 2000: 53: 101-5.

3. Tokuchi Y, Kamachi M, Harada M, Hasegawa M, Mishina T, Yamashiro K, et al. Synchronous triple lung cancers after treatment for non-Hodgkin's lymphoma: metachronous quadruple cancers. Intern Med 2003; 42: 1031-4.
4. Nishino R, Daga H, Sasaki R, Moritani C, Ohashi $\mathrm{N}$, Arita K, et al. A case of severe pneumoconiosis with synchronous triple lung cancer. Nihon Kokyuki Gakkai Zasshi 2003; 41: 491-5.

5. Brun S, Paparelli C, Sinnona N, Venuti VM. Synchronous lung cancer; clinical case of triple lung carcinoma. G Chir 2002; 23: 43-4.

6. Motohiro A, Matsumoto T, Ienaga S. Synchronous growth of triple lung cancer. Surg Today 1995; 25: 1054-6.

7. Hoshi E, Aoyama K, Takayanagi N. A case of a synchronous triple primary lung cancer with hamartoma. Kyobu Geka 1995; 48: 251-5.

8. Dalton ML, Warner RL. Triple synchronous primary lung carcinomas treated with simultaneous resection. J Med Assoc Ga 1991; 80: 287-90.

9. Badiali P, Alloisio M, Lombardi L. Synchronous triple carcinoma of the lung in one patient. Tumori 1987; 73: 525-9.

10. Sato K, Maekawa T, Yabuki K, Tamasaki Y, Maekawa H, Kudo K, et al. A case of triple synchronous cancers occurring in the gallbladder, common bile duct, and pancreas. J Gastroenterol 2003; 38: 97-100.

11. Tamura M, Shinagawa M, Funaki Y. Synchronous triple early cancers occurring in the stomach, colon and gallbladder. Asian J Surg 2003; 26: 46-8.

12. Chang YT, Tsai CI, Yang TH, Shih CW, Wu MS, Lin JT. Synchronous triple cancers at middle and lower esophagus and stomach with different histological feature and genetic alterations. J Gastroenterol Hepatol 2002; 17: 724-7.

13. Chen JH, Chen CC, Tzeng LM, Tsay SH, Chiang $\mathrm{JH}$, Lu CC, et al. Resection of triple synchronous tumors: gastric adenocarcinoma, gallbladder adenocarcinoma and stroma tumor of the stomach. Zhonghua Yi Xue Za Zhi 2001; 64: 655-60.

14. Isin Dogan Ekici A, Kucukali T, Coskun Salman M, Ayhan A. Triple simultaneous primary gynaecological malignancies in a 56-year-old patient. Int J Gynecol Cancer 2006; 16: 1947-50.

15. Jun SY, Cho KJ, Kim CS, Ayala AG, Ro JY. Triple synchronous neoplasms in one kidney: report of a case and review of the literature. Ann Diagn Pathol 2003; 7: 374-80.

16. Satoh H, Momma T, Saito S, Hirose S. A case of synchronous triple primary carcinomas of the kidney, bladder and prostate. Hinyokika Kiyo 2003; 49: 261-4. 
17. Takada T, Honda M, Momohara C, Komori K, Fujioka H. Synchronous triple urogenital cancer (renal cancer, bladder cancer, prostatic cancer): a case report. Hinyokika Kiyo 2002; 48: 239-42.

18. Vallejo Herrador J, Sanchez de la Muela P, Diz Rodrigez R, Martin-Laborda F. Synchronous primary urologic triple neoplasia. Report of a new case and review of the literature. Actas Urol Esp 2002; 26: 57-9.

19. Harima M, Narita K, Kobayakawa H, Tsujino T, Yamamoto S, Fukushima S, et al. A case of synchronous triple primary cancers of prostate, kidney and bladder. Hinyokika Kiyo 1998; 44: 675-8.

20. Tamura K, Inoue K, Fukata S, Kamada M, Shuin T. Small cell carcinoma of the urinary bladder with synchronous esophageal cancer and incidental lung cancer: a case report. Hinyokika Kiyo 2001; 47: 273-6.

21. Patel S, Alfonso AE. Landis J, Suarez J. Three synchronous multiorgan primary cancers. All stage I. Arch Surg 1985; 120: 1182-4.

22. Taira K, Shiraishi M, Sunagawa H, Takushi Y, Shimoji H, Tomita S, et al. Resection of triple synchronous cancers: a case report. Hepatogastroenterology 1999; 46: 199-203.

23. Okajima E, Ozono S, Nagayoshi J, Uemura $H$, Hirao Y, Nakajima Y, et al. A case report of synchronous triple cancer resected simultaneously. Jpn J Clin Oncol 1994; 24: 166-70.

24. Kurihara T, Ishida T, Miyamoto Y, Mishima T, Suda A, Izuo M. A case of quartet cancer: a carcinoma of the breast followed by three synchronous cancers (kidney, thyroid and colon). Gan No Rinsho 1989; 35: 955-62. 